\title{
Modelling the Effect of Carbon Content on Hot Strength of Steels Using a Modified Artificial Neural Network
}

\author{
L. X. KONG, P. D. HODGSON and D. C. COLLINSON ${ }^{1)}$
}

School of Engineering and Technology, Deakin University, Geelong, Vic 3217, Australia. 336 Glenferrie Rd., Malvern, Vic 3144, Australia.

1) Ajax Technology Centre,

(Received on April 6, 1998; accepted in final form on June 14, 1998)

\begin{abstract}
The hot strength of austenitic steels with the carbon content varying from 0.0037 to 0.79 wt\% was modelled using artificial neural networks (ANN). The carbon content has a complex effect on flow strength of austenite. An increase in carbon content reduces the flow stress of the steels at high temperatures and low strain rates, while it increases the flow stress at low temperatures and high strain rates, especially at low strains. In addition, increasing carbon to above $0.4 \mathrm{wt} \%$ dramatically reduces the peak strain for the initiation of dynamic recrystallisation at high Zener-Hollomon parameter, $Z$. Given the complexity of the deformation and recrystallisation behaviours of these steels, no phenomenological or simple empirical models are able to predict the flow stress over the full carbon range. In this work, the back error propagation algorithm of the ANN model with one hidden layer bias was used, with the number if hidden nodes optimised. The data up to a strain of 4 were used to predict the strength in both work hardening and dynamic recrystallisation regimes. The training speed was an important parameter and was optimised by trimming the data set and learning procedures. The effects of the carbon content on flow stress, peak strains and peak stresses observed from the experiment were accurately represented. However, it was found that the training data set also needed to be optimised to accurately predict the hot strength of the steels.
\end{abstract}

KEY WORDS: hot strength; artificial neural networks; austenitic steel; model optimisation.

\section{Introduction}

Control of the thickness of steel strip requires accurate prediction of the rolling load which, in turn, requires prediction of the flow strength. ${ }^{1)}$ For steels of a given composition, a number of models, such as the phenomenological model, ${ }^{2-4)}$ empirical model, ${ }^{5)}$ finite-element method $^{6)}$ and artificial neural network (ANN) model, ${ }^{1,7)}$ have been developed to investigate the effects of the deformation conditions-temperature, strain and strain rate-on the hot strength. However, the influence of carbon content has not been extensively studied partly, because many authors have reported little or no effect of the carbon content on the flow stress of austenitic steels. ${ }^{8}{ }^{8}$ Recent work, though, has shown that the carbon causes a significant variation in the steel strength under certain processing conditions. ${ }^{8-10)}$

Collinson et $_{\text {al }}{ }^{8-10)}$ studied the effect of carbon content on the flow strength, static and dynamic recrystallisation behaviour of $\mathrm{C}-\mathrm{Mn}$ steels with carbon contents ranging from 0.0037 to $0.79 \mathrm{wt} \%$. It was observed that the carbon content had a complex effect on the flow strength of austenite. Increasing carbon reduced the flow strength by up to $30 \%$ under conditions of low strain rates and high temperatures. At the high strain rates and low temperatures typical of hot rolling, increasing carbon resulted in increases in the flow stress of over $100 \%$ at low strains. The results showed that there were also significant effects of carbon on the dynamic recrystallisation behaviour. Under conditions of multipeak dynamic recrystallisation in the low carbon steels, the addition of carbon reduced both the amplitude and duration of the cycling. Under single peak dynamic recrystallisation conditions, the low carbon steel showed a continuous increase in the critical strain for the initiation of dynamic recrystallisation as the strain rate of testing was increased and the temperature reduced. For the high carbon steel the critical strain for dynamic recrystallisation increased up to a strain 0.7 and then remained constant, despite further reductions in the temperature or increases in the strain rate.

Because of the complex effect of carbon content on flow strength discussed above, no simple physical model has been developed to include the influence of both chemical composition and deformation conditions. With artificial neural networks, Hwu et al. ${ }^{7)}$ investigated the constitutive behaviour of carbon steels, including the effects of carbon content, and indicated that the prediction of flow strength with ANN models had high accuracy. In the present research, the effect of chemical compositions on flow strength of austenitic steels model was investigated with a supervised back error propagation ANN modified with information from an integrated phenomenological and artificial neural network (IPANN) 
Table 1. Compositions of the steels used.

\begin{tabular}{|c|c|c|c|c|c|c|c|c|c|c|}
\hline Steel & Heat no. & $\mathrm{C}$ & $\mathrm{Mn}$ & $\mathrm{Ni}$ & $\mathrm{Cu}$ & $\mathrm{Cr}$ & $\mathrm{Mo}$ & $\mathrm{V}$ & $\mathrm{C}_{\text {rq }}$ & $\mathrm{Q}_{\alpha<}{ }^{(2)}(\mathrm{kJ} / \mathrm{mol})$ \\
\hline $\mathrm{A}$ & $\mathrm{V} 3598$ & 0.0037 & 1.00 & 0.006 & 0.003 & 0.005 & 0.003 & 0.003 & 0.1732 & 295 \\
\hline B & V3435 & 0.017 & 0.98 & 0.008 & 0.004 & 0.003 & 0.003 & 0.003 & 0.1829 & 323 \\
\hline C & V3373 & 0.065 & 0.99 & 0.007 & 0.003 & 0.004 & 0.002 & 0.003 & 0.2325 & 323 \\
\hline D & V3374 & 0.21 & 0.99 & 0.007 & 0.003 & 0.003 & 0.002 & 0.003 & 0.3773 & 305 \\
\hline E & V3375 & 0.41 & 1.00 & 0.006 & 0.003 & 0.003 & 0.002 & 0.003 & 0.5789 & 257 \\
\hline F & V3376 & 0.58 & 1.06 & 0.006 & 0.003 & 0.003 & 0.002 & 0.003 & 0.7589 & 256 \\
\hline G & V3377 & 0.79 & 1.04 & 0.006 & 0.003 & 0.003 & 0.002 & 0.003 & 0.9655 & 244 \\
\hline H & V3473 & 0.085 & 0.32 & 0.003 & 0.003 & 0.003 & 0.002 & 0.003 & 0.1405 & 320 \\
\hline I & V3474 & 0.085 & 1.69 & 0.004 & 0.003 & 0.003 & 0.002 & 0.003 & 0.3691 & 320 \\
\hline
\end{tabular}

model developed by Hodgson et al. ${ }^{1)}$ and an empirical model. ${ }^{5)}$ A selection criterion for the training data has been developed to optimise the selection of training data set and accurately interpolate and predict the flow stresses corresponding to the conditions within the boundary training conditions.

\section{Experimental Results}

The nine heats used in this work were based on electrolytic iron with carbon additions from 0.0037 to $0.79 \mathrm{wt} \%$ and similar levels of manganese and other materials with exception to the last two heats which have different manganese from other heats (Table 1) to evaluate the effect of manganese. The steels were cast in a vacuum furnace as $65 \mathrm{~kg}$ ingots before being hot rolled, machined into torsion samples and torsion tested using the hot torsion machine at BHP, Australia. The austenite grain size prior to testing was close to $100 \mu \mathrm{m}$ for all steels and tests were performed at temperatures of 900 , 1000 and $1100^{\circ} \mathrm{C}$ and at strain rates from 1 to $100 \mathrm{~s}^{-1}$. Further details on the experiments and sampling were previously reported. ${ }^{8)}$

The stress-strain behaviour of steels A, C, E and G at a temperature of $900^{\circ} \mathrm{C}$ and strain rates of 1,10 and $100 \mathrm{~s}^{-1}$ is shown in Fig. 1. The stress-strain behaviour is typical of steels deformed in the austenite phase region showing work hardening up to a peak stress followed by softening, indicative of dynamic recrystallisation. The stress increases with an increase in strain rate and decrease in temperature for all steels as expected. However, the carbon content presents a very complex effect on stress-strain curves, as discussed elsewhere. ${ }^{8-10)}$ At a low strain rate of $1 \mathrm{~s}^{-1}$, the stress decreases with an increase in carbon content while at a high strain rate of $100 \mathrm{~s}^{-1}$, increasing carbon content leads to constantly increasing stress (Fig. 1). At the strain rate of $10 \mathrm{~s}^{-1}$, the stress increases when carbon is varied from 0.0037 to $0.065 \mathrm{wt} \%$, but decreases with carbon increasing to $0.41 \mathrm{wt} \%$ and then increases when carbon increases to $0.79 \mathrm{wt} \%$. However, the variation in stress caused by the increase in carbon content is small compared with those caused by the variation of temperature and strain rate.

The effects of strain rate and temperature have been (a)

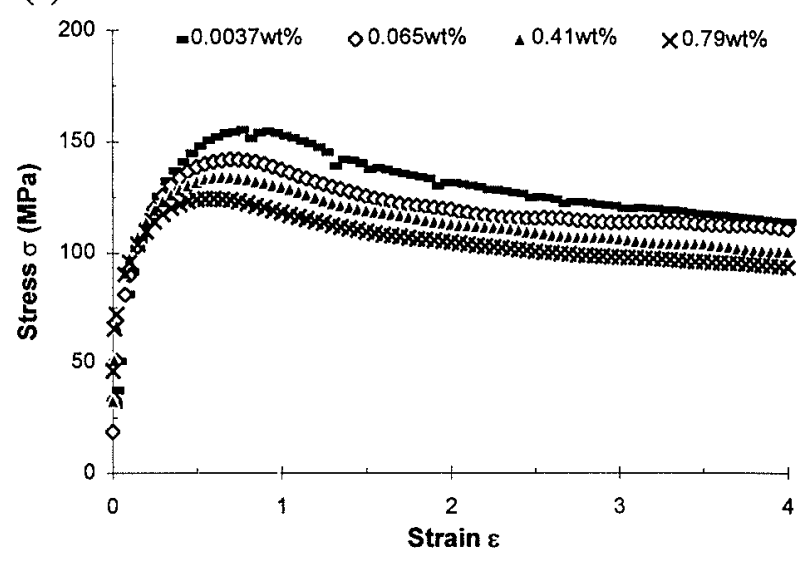

(b)

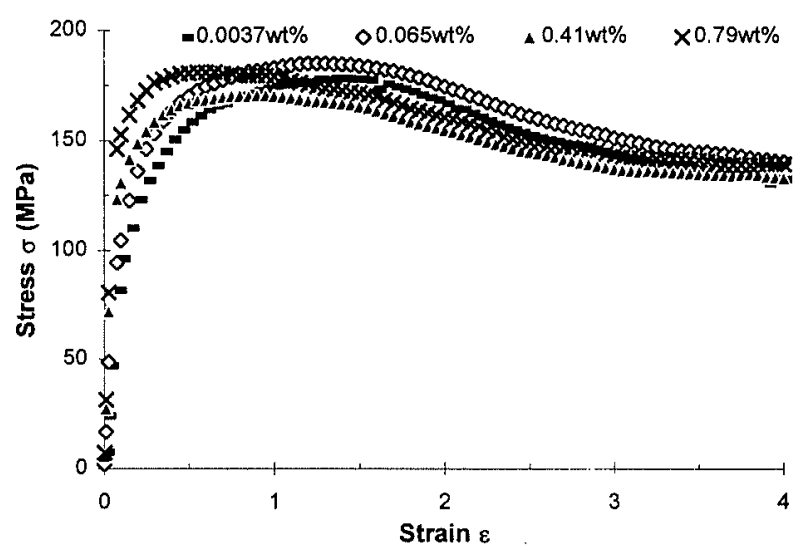

(c)

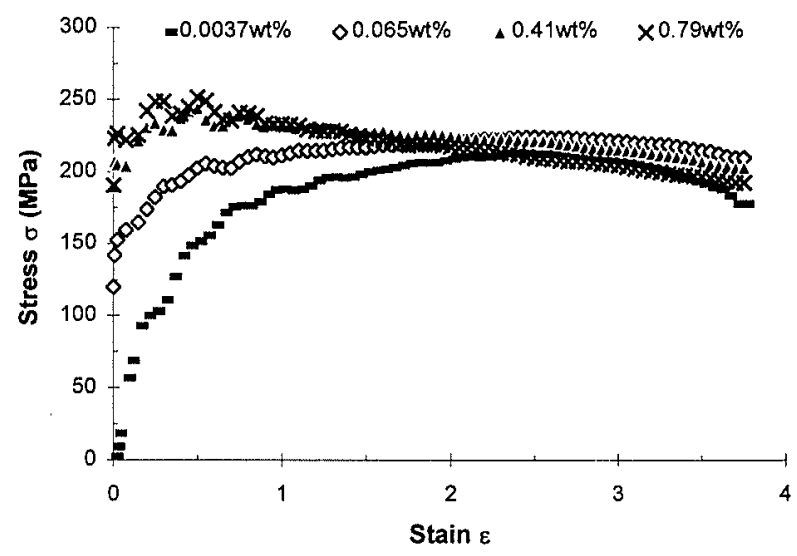

Fig. 1. Measured stress--strain curves at a temperature of $900^{\circ} \mathrm{C}$.

Strain rate of (a) $1 \mathrm{~s}^{-1}$, (b) $10 \mathrm{~s}^{-1}$, (c) $100 \mathrm{~s}^{-1}$.

discussed by Collinson et al. ${ }^{8-10)}$ and their contribution was analysed through the Zener-Hollomon parameter:

$$
Z=\dot{\varepsilon} \exp \left(Q_{\text {def }} / R T\right)
$$

where $Q_{\text {def }}$ is the activation energy with values listed in Table 1 for steels of different carbon contents, $\dot{\varepsilon}$ is the strain rate, $T$ is the temperature and $R$ is gas constant. Although the peak stress generally increases with an increase in the Zener-Hollomon parameter, $Z$, for the same steel (Fig. 2), no simple trend can be developed for all of the steels studied in this work due to the carbon 


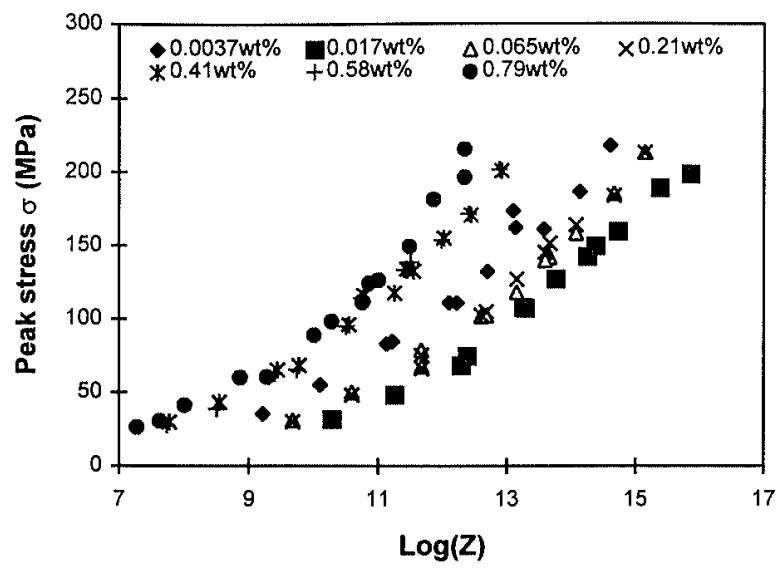

Fig. 2. Peak stress plotted against Zener-Hollomon parameter for all steels.

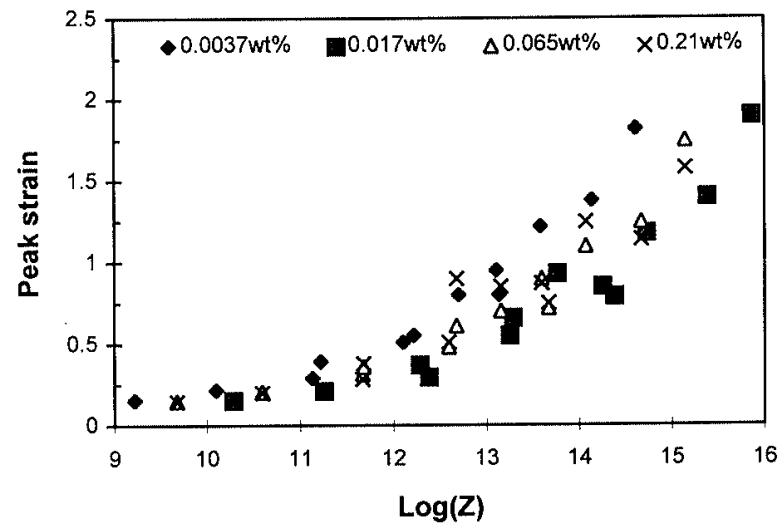

(a)

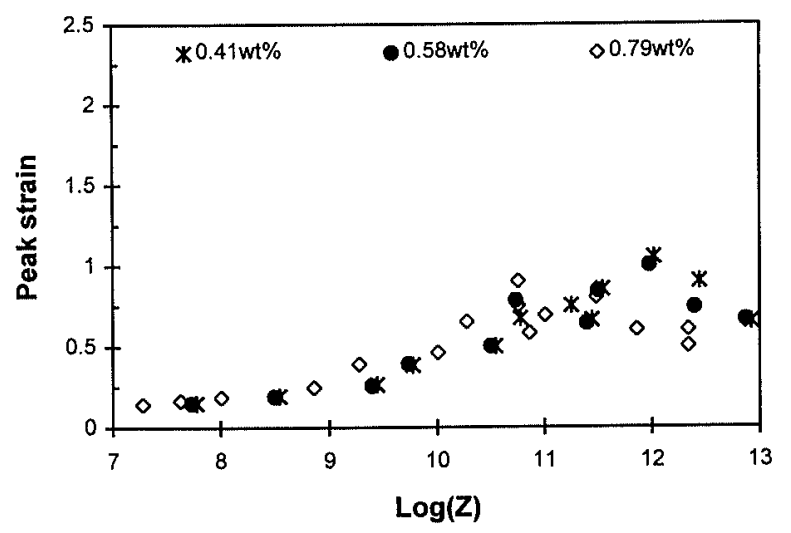

(b)

Fig. 3. Variation of peak strain with Zener-Hollomon parameter for all steels, (a) low carbon (b) medium to high carbon.

content leading to the variation of $Z$ parameter as the activation energy $Q_{\text {def }}$ varies from $244 \mathrm{~kJ} / \mathrm{mol}$ at the high carbon content to around $323 \mathrm{~kJ} / \mathrm{mol}$ for low carbon steels. ${ }^{10)}$

The increase in carbon content tends to shift the peak strain to a lower value, particularly for high $Z$; that is at lower temperatures and higher strain rates. For lower carbon steels (up to $0.21 \mathrm{wt} \%$ ), the peak strain increases with increasing $Z$ (Fig. 3) due to the lower recovery rates delaying the onset of dynamic recrystallisation. ${ }^{9)}$

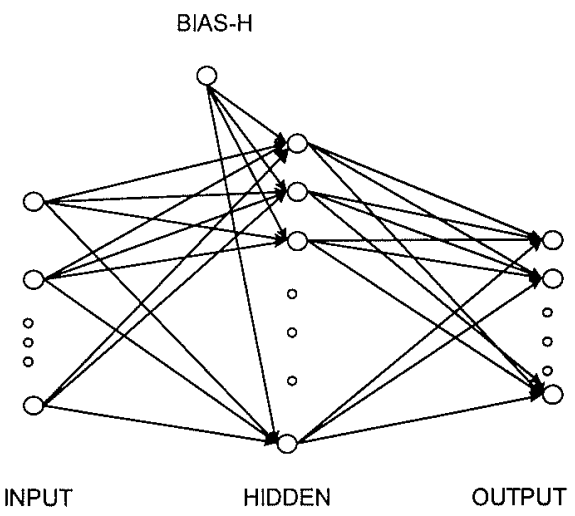

Fig. 4. Multilayer perceptron neural network.

However, this behaviour is not observed for the steels with higher carbon contents. For steels of carbon higher than $0.41 \mathrm{wt} \%$, the peak strain gradually increases with the $Z$ until a value of $10^{11}$ is reached. After that, the strain corresponding to peak stress appears to reduce slightly with a further increase in $Z$.

From the above, it is apparent that there are complex effects on both the peak stress and strain and a pronounced effect on the stress-strain ${ }^{10)}$ with respect to $Z$ with changing carbon. Increasing carbon lowers the flow stress at low strain rates and high temperatures (low $Z$ ) but increases it at high strains and low temperatures (high $Z$ ). Based on this, the development of a mathematical model to cover the whole range of effects is difficult.

\section{Artificial Neural Networks}

\subsection{Multilayer ANN Perceptron}

The artificial neural network used in the current work is a supervised multilayer feedforward network trained with the standard backpropagation algorithm. The scheme of a three-layer network with one hidden layer and an offset bias to the hidden layer is shown in Fig. 4. There are two important characteristics of this multilayer perceptron. First, its processing elements are nonlinear. The nonlinearity function must be smooth with the logistic function; the hyperbolic tangent being the most widely utilised. Second, the processing elements are massively interconnected such that any element of a given layer feeds all the elements of the next layer.

The multilayer perceptron are trained with error correction learning or the error backpropagation algorithm. The equation to update the weights in momentum learning is: ${ }^{11)}$

$$
w_{i j}(n+1)=w_{i j}(n)+\eta \delta_{i}(n) x_{j}(n)+\alpha\left(w_{i j}(n)-w_{i j}(n-1)\right)
$$

where $w_{i j}(n)$ is the weight between nodes $i$ and $j$ at iteration $n, \delta_{i}(n)$ is the local error which can be directly computed from $e_{i}(n)$, the instantaneous error between desired response $d_{i}(n)$ and system response $y_{i}(n)$, at the output processing elements or as a weighted sum of errors at the internal processing elements, $\eta$ is step size, and $\alpha$ is the momentum and is set between 0.1 and 0.9 .

The selection of training algorithm, stopping criteria 
and representative training set is the most important and practical aspect related to training an ANN model. The total training iteration number and the cross-validation were specified and the training stopped once either the iteration number or the mean square error of the test set reached their prescribed values. The mean square error of the training set was then analysed to evaluate the performance of the training.

\subsection{Model Development}

ANN models have been applied successfully to predict the flow stress of hot deformed steels ${ }^{1)}$ with varying carbon content. ${ }^{7)}$ However, due to the limited experimental data available in that work and the ANN models used, the ANN could not be used to generalise a model to predict the effects of a wide range of processing parameters and material compositions. As indicated by Hodgson et al.," the understanding of the physical principals involved in the process greatly improves the predictions of ANNs. In this work, the parameters derived from the physical models are designated as inputs of the ANN model in addition to the processing parameters strain, strain rate and temperature, and the composition parameter carbon content. The predictions of this model were compared with those of an ANN model based on only the processing and composition parameters as inputs. The carbon equivalent ${ }^{7,12)}$ was used here as an index to evaluate the effect of compositions:

$$
C_{\text {eq }}=C+\frac{\mathrm{Mn}}{6}+\frac{\mathrm{Ni}+\mathrm{Cu}}{15}+\frac{\mathrm{Cr}+\mathrm{Mo}+\mathrm{V}}{5}
$$

and the effect of manganese and other materials could be predicted at a reasonable accuracy for steels such as $\mathrm{H}$ and $\mathrm{I}$.

The work hardening coefficient, defined as the slope of the stress-strain curve $\left.\left.(\theta=\partial \sigma / \partial \varepsilon)\right|_{\dot{\varepsilon}, T}\right)$, is an important aspect of stress-strain behaviours for steels with varying carbon content. the primary influence of the carbon content is due to its effect in the work hardening rate. ${ }^{9)}$ The work hardening rate varies with stress differently for four steels deformed at a strain rate of $1 \mathrm{~s}^{-1}$ and a temperature of $900^{\circ} \mathrm{C}$ (Fig. 5). Although it varies in a similar way for the steels with carbon up to $0.41 \%$, the increase in carbon to $0.79 \%$ greatly increases the stress and the work hardening rate as the peak strain decreases and the peak stress increases.

According to the Kocks and Mecking (KM) model, ${ }^{2,13 \text { ) }}$ there is a linear relationship between the work hardening coefficient and the flow stress in most of work hardening regime,

$$
\theta=\theta_{0}\left(1-\frac{\sigma}{\sigma_{\mathrm{s}}}\right)
$$

where $\theta_{0}=\alpha G b k_{1} / 2$, which is independent of strain rate and depends on temperature through the temperature dependence of $G$, and $\sigma_{\mathrm{s}}=\alpha G b\left(k_{1} / k_{2}\right)$ which is the saturation stress in the absence of dynamic recrystallisation. Estrin and Mecking further modified the KM model and established, ${ }^{3)}$

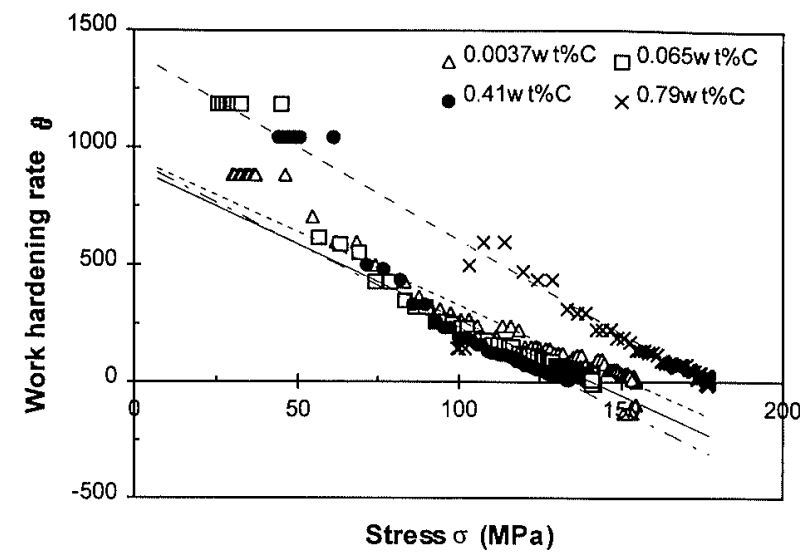

Fig. 5. Variation of work hardening rate $v s$. stress with carbon content for steels deformed at $1 \mathrm{~s}^{-1}$ and $900^{\circ} \mathrm{C}$.

Table 2. Scheme of ANN models.

\begin{tabular}{|c|c|c|c|}
\hline Scheme & Input layer & Hidden layer & Output layer \\
\hline 1 & $\begin{array}{c}\operatorname{strain}(\varepsilon), \operatorname{strain} \operatorname{rate}(\dot{\varepsilon}), \text { temperature }(T), \\
\operatorname{carbon}\left(C_{e q}\right)\end{array}$ & 1 & stress $(\sigma)$ \\
\hline 2 & $\varepsilon, \dot{\varepsilon}, T, C_{e q}, \log (Z)$, work hardening \\
$\operatorname{rate}(\theta), \sqrt{\theta \sigma}$ & 1 & stress $(\sigma)$ \\
\hline
\end{tabular}

$$
\theta \sigma=A-B \sigma^{2}
$$

which after integration becomes,

$$
\sigma=\left[\sigma_{\mathrm{s}}^{2}+\left(\sigma_{0}^{2}-\sigma_{\mathrm{s}}^{2}\right) \exp (-2 B \varepsilon)\right]^{1 / 2}
$$

where $\sigma_{0}$ is initial stress when $\varepsilon=0$ and $A, B$ are constants which are a function of Zener-Hollomon parameter defined in Eq. (1). Incorporating the work hardening coefficient $(\theta)$ and the square root product of $\theta$ and $\sigma(\sqrt{\theta \sigma})$ with the ANN model gives the IPANN model. ${ }^{1)}$ The IPANN model improved the accuracy of its predictions comparing to the ANN model for an austenitic stainless steel. As the activation energy $Q_{\text {def }}$ is different for steels, the Zener-Hollomon parameter defined in Eq. (1) is a parameter able to reflect the effect of activation energy and deformation. The ANN schemes employed in this work are listed as Table 2. The inputs used in Scheme 2 are three more than conventional Scheme 1 and provide much more information. It needs to be noted that the experimental data must be analysed to obtain $Z, \theta$ and $\sqrt{\theta \sigma}$ for the training and test data from the physical models.

\subsection{Training Data Preparation}

It is necessary to have a sufficient database and develop a suitable model to generalise a model based on the ANN method. The less training data used, the higher the agreement with the training data but the worse the prediction of the test data not used for training. ${ }^{1)}$ However, although an increase in the training data improves the generalisation of the ANN model, it will simultaneously increase the time required to train the model.

The preparation of the training data set is related to the way the outputs vary with inputs and the availability of experimental data. If the output varies with inputs in 


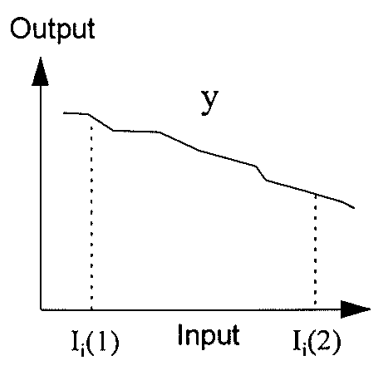

(a)

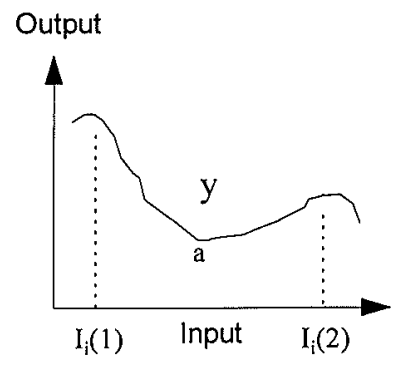

(b)
Fig. 6. Strategy of training data preparation (a) output varying in a simple way, (b) output varying in a complex way.

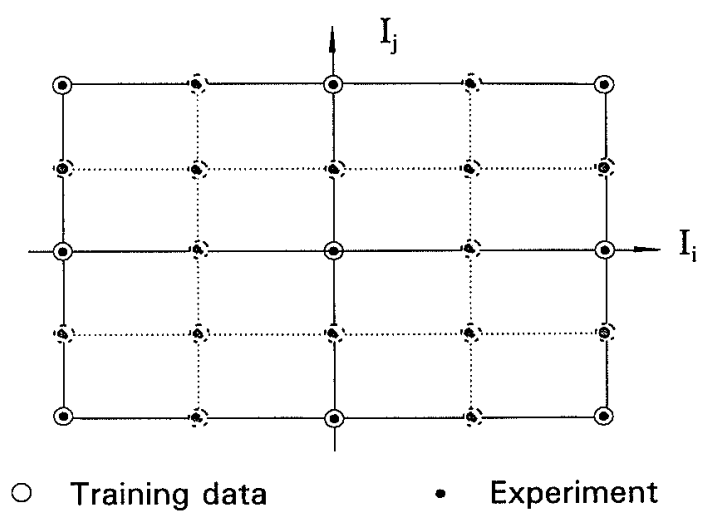

Fig. 7. Two-dimensional analysis of training data.

different ways as shown in Fig. 6, the training data used to generalise a model should be prepared differently. Suppose that there are the same values for the output at two points $I_{i}(1)$ and $I_{i}(2)$ but one varies in a simple trend or can be described with a simple mathematical formula (Fig. 6(a)) while the other varies in a complicate way (Fig. 6(b)). Obviously, it requires fewer training data for the former to create an accurate model than the latter.

For the outputs which vary as in Fig. 6(b), it is necessary to optimise the training data used, as too small a training set will not give a generalised model while too large a training set will greatly increase the time for training. This optimisation process will reduce the experimental effort to obtain the data being used for training and improve the prediction accuracy and model generalisation. Figure 7 outlines a scheme for a twodimensional (two independent inputs) model. If the outputs vary with inputs $I(i)$ and $I(j)$ in a relatively simple pattern, (Fig. 6(a)), two boundary positions and one intermediate position for every input are sufficient to generalise an ANN model as shown in Fig. 7 as solid points. However, for outputs which are more complex function of the inputs, (Fig. 6(b)), the training data require more points, for example, including those of dashed points (Fig. 7). Although evenly distributed experimental data will generally be acceptable as training data for most cases, a problem dependent scheme is required if the outputs vary in a complex way and there is previous knowledge on the problem as shown in Fig. 6(b) for point $a$.

Therefore, total data used for training an ANN model with $n$ independent inputs will be,

$$
m=\sum_{i_{n}=1}^{N_{n}} \sum_{i_{n}-1=1}^{N_{n-1}} \cdots \sum_{i_{1}=1}^{N_{1}} \delta_{I_{1}\left(i_{1}\right), I_{2}\left(i_{2}\right), \cdots, I_{n}\left(i_{n}\right)}
$$

where

$$
\delta_{I_{1}\left(i_{1}\right), I_{2}\left(i_{2}\right), \cdots, I_{n}\left(i_{n}\right)}=\delta_{I_{1}\left(i_{1}\right)} \delta_{I_{2}\left(i_{2}\right)} \cdots \delta_{I_{n}\left(i_{n}\right)}
$$

and defined as,

$$
\begin{aligned}
& \delta_{I_{1}\left(i_{1}\right), I_{2}\left(i_{2}\right), \cdots I_{n}\left(i_{n}\right)} \\
& \quad= \begin{cases}1 & \text { If the point is used in the training data set } \\
0 & \text { If the point is not used in the training data set }\end{cases}
\end{aligned}
$$

$I_{i}$ is a independent input and $N_{i}$ is the maximum points used for the independent input $i$. Obviously, if every point of all independent inputs is used in the training data set, the total data used will be,

$$
m=N_{1} N_{2} \cdots N_{n}
$$

Hence, there are two main approaches to optimise the training data. One is to reduce the independent input number, while the other is to appropriately select the representative points for every independent input. In this work, there are four independent inputs: strain, strain rate, temperature, equivalent carbon content and seven different carbon contents of which four used for training the model while three for test. There are three strain rates and three temperatures in the experiment.

\section{ANN Predictions}

\subsection{Prediction on Steels with a Given Carbon Content}

The ANN models of two different schemes discussed above were initially used to predict the stress-strain behaviour of steels with a constant carbon content. The $0.21 \mathrm{wt} \%$ carbon steel deformed at strain rates of 1,10 and $100 \mathrm{~s}^{-1}$ and temperatures of 900,1000 and $1100^{\circ} \mathrm{C}$ was used, with the number of training data set being 738 (as calculated with Eq. (10)).

The relative errors of the same training data with two ANN schemes are shown in Fig. 8. Although both schemes predict the flow stress with a high level of accuracy, scheme 2 incorporating work hardening rate and the logarithm of the Zener-Hollomon parameter as inputs is more accurate in both the work hardening and dynamic recrystallisation regimes. This can be seen from the error analysis as the conventional ANN has an average error of $0.54 \%$ and a standard error ${ }^{1)}$ of $7 \%$ while these two coefficients are 0.2 and $3.9 \%$ respectively for the modified ANN model. The relative errors of most of the training data for Scheme 2 are within $\pm 5 \%$. Higher error values were observed at the lowest strain for all steels because the ANN model is unable to predict the steep variation in stress with strain. ${ }^{1)}$ This is further complicated for steels deformed at a strain rate of $100 \mathrm{~s}^{-1}$. The current torsion machine configuration includes a shock wave which shows up an oscillation in the stressstrain curve at high strain rates. Due to experimental noise, the variation of stress with strain or the work hardening rate in this region is changing frequently and prediction with the ANN models is therefore less ac- 


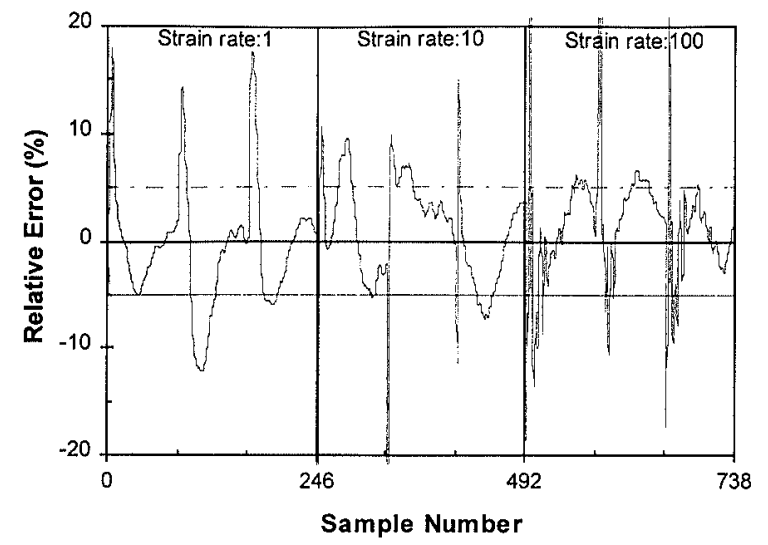

(a) Conventional ANN (Scheme 1)

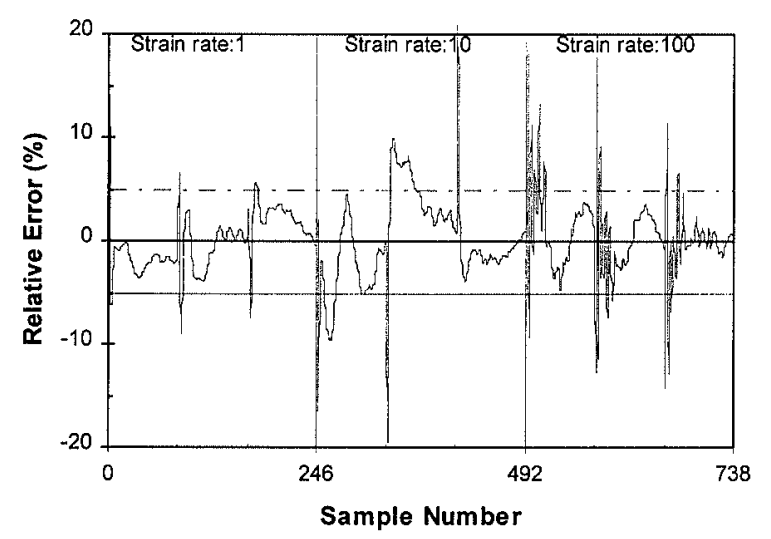

(b) Modified ANN (Scheme 2)

Fig. 8. Relative error with two training schemes for a $0.21 \mathrm{wt} \%$ carbon content.

curate. To accurately predict the flow strength, therefore, the analysis of training data is every important as experimental noise will provide fault information. This is one weakness of the ANN model over physical or empirical models and will be particularly important for online prediction and process control.

The trained model of scheme 2 was tested with experimental data not used for training. The predicted flow stresses of the test data are shown in Fig. 9 and compared with measured results. As sufficient points were provided with the training data in particular for strain, the prediction is almost as accurate as training. Based on this, the strain is not used in the further predictions as a variable to test the training. It is observed that both the peak stress and strain to peak stress for all deformation conditions are generally accurately predicted, with only a few peak stresses having a significant error. The prediction in dynamic recrystallisation regime is not as accurate as this in work hardening area. This is partly because simple phenomenological model covering only work hardening was incorporated with the current model.

The improvement in prediction with the modified ANN model can also be seen from the analysis of sensitivity of stress to changes in the input parameters (Table 3). The sensitivity, which reflects the effect that

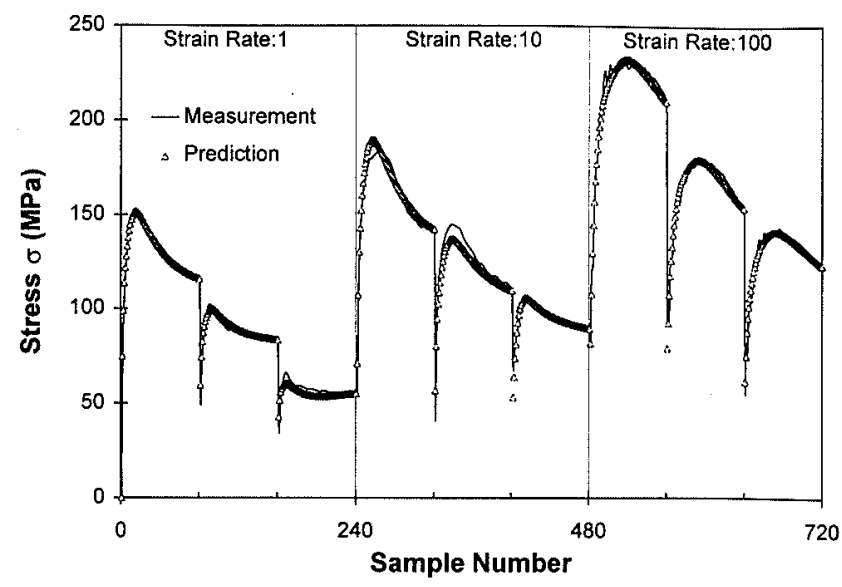

Fig. 9. Comparison of ANN prediction (scheme 2) with experiment using test data $(0.21 \mathrm{wt} \% \mathrm{C})$.

Table 3. Sensitivity comparison of two schemes.

\begin{tabular}{|l|c|c|c|c|c|c|}
\hline & $\log (z)$ & $\theta$ & $\dot{\varepsilon}$ & $\varepsilon$ & $\sqrt{g_{\sigma}}$ & $T$ \\
\hline Scheme 1 & & & 0.46 & 0.3 & & 0.24 \\
\hline Scheme 2 & 0.305 & 0.3 & 0.13 & 0.11 & 0.11 & 0.045 \\
\hline
\end{tabular}

each of the network inputs is having on the network output, is calculated by summing the changes in the output variables caused by moving the input variables by a small amount over the entire sensitivity calculation dataset. Of the six inputs used, the logarithm of the Zener-Hollomon parameter $(\log (Z))$ and the work hardening rate $(\theta)$ were the two most sensitive variables, with a sensitivity of 0.305 and 0.3 , respectively, in comparison with 0.045 for temperature and 0.11 for strain. However, if temperature or strain rate were not included as inputs, the prediction became noticeably worse. Since there was an improvement with the modified ANN model for all predictions in the current work, the following sections will only discuss the predictions with the modified ANN model (scheme 2) and the conventional ANN will not be considered.

\subsection{Prediction on Training Data without Intermediate Information}

The experimental data from steels A, C, E and G were used as training data with strains up to 4 , strain rates of 1 and $100 \mathrm{~s}^{-1}$, and temperatures of 900 and $1100^{\circ} \mathrm{C}$ while the data at the strain rate of $10 \mathrm{~s}^{-1}$ and the temperature of $1000^{\circ} \mathrm{C}$ were removed. The predicted stresses from the training data are compared with measured data in Fig. 10. In the seven inputs used, the work hardening rate is the most sensitive parameter with a sensitivity of 0.358 compared with 0.064 for the carbon content. The average error is $-0.6 \%$ and the standard error is $5.2 \%$. As the prediction here has one more independent input, carbon content, both the average and standard errors are higher than the earlier result with a constant carbon content of 0.21 wt \% (Fig. 8(b)).

Since only the boundary information of strain rate and temperature were provided, the prediction of flow stress on test data of different strain rate and/or temperature exhibited obvious different characteristics. Figure 11 shows the comparison of predicted and experimental 


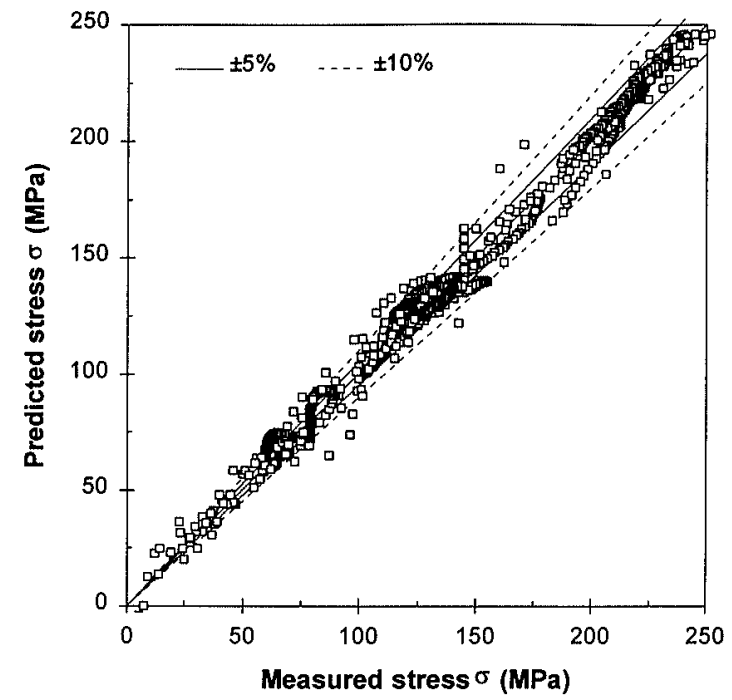

Fig. 10. Comparison of predicted and measured stress using only training data for steels $\mathrm{A}, \mathrm{C}, \mathrm{E}$ and $\mathrm{G}$ (with boundary information only).

stresses on test data with at least one of the three independent inputs (carbon content, strain rate and temperature) different from the training data. If only the temperature of the test data varies from the training data while the strain rate remains constant, the prediction is very accurate. However, if the strain rate in the test data is different from that used in the training data set, the prediction greatly underestimates the stress (Figs. 11(a) and $11(\mathrm{c})$ ). In addition, carbon content does not have significant influence on the prediction (Fig. 11) which may be described from its low sensitivity of 0.064 .

The different effects of strain rate and temperature can be explained in two ways. First, stress varies in a complicated way with the strain rate and the carbon content (Fig. 1), as discussed previously. In contrast, the stress almost decreases linearly with an increase in both temperature and carbon (Fig. 12) if the strain rate keeps constant. Secondly, the strain rates used in the training represent a very large change and the information provided with the boundary conditions of strain rate are not sufficient to develop a generalised model to cover the complicated relationship between stress and strain rate.

\subsection{Prediction with Full Training Data of Strain Rate and Temperature}

As the objective of this work was to predict the stress-strain behaviour of steels with a varying carbon content, the full experimental data set of steels A, C, E and $\mathrm{G}$ was used to predict the behaviour of other steels. In this case, the total training points used were $82 \times 4 \times$ $3 \times 3$, that is, 82 points for strain, four steels, three temperatures and three strain rates.

The prediction on the training data is shown in Fig. 13 with the average error of $1.7 \%$ and standard error of $6.3 \%$. The average and standard errors increase with an increase in the number of independent inputs and training data compared with those two errors for all three predictions in Figs. 8(b), 10 and 13. Therefore, model accuracy is sacrificed with the incorporation of more information and more generalisation.

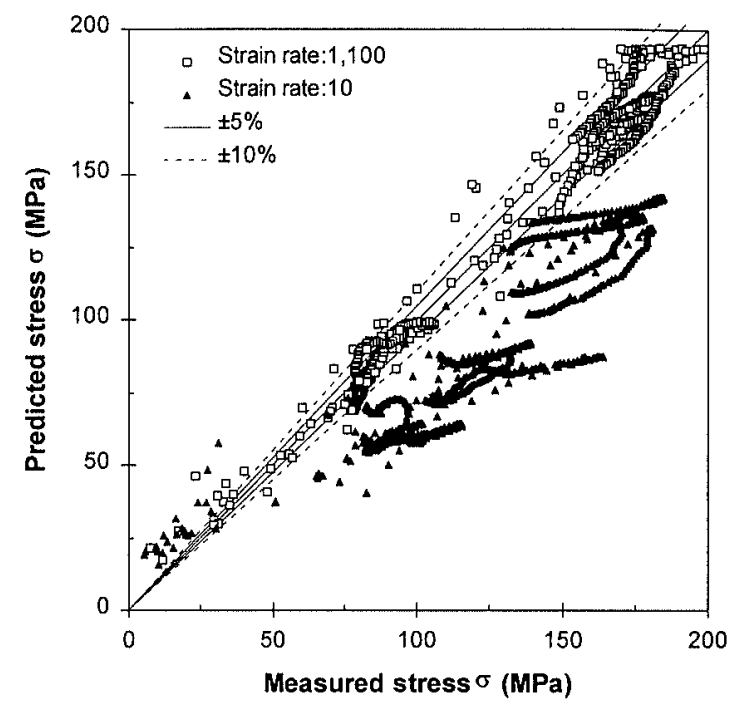

(a) Same steels but different strain rates and temperatures

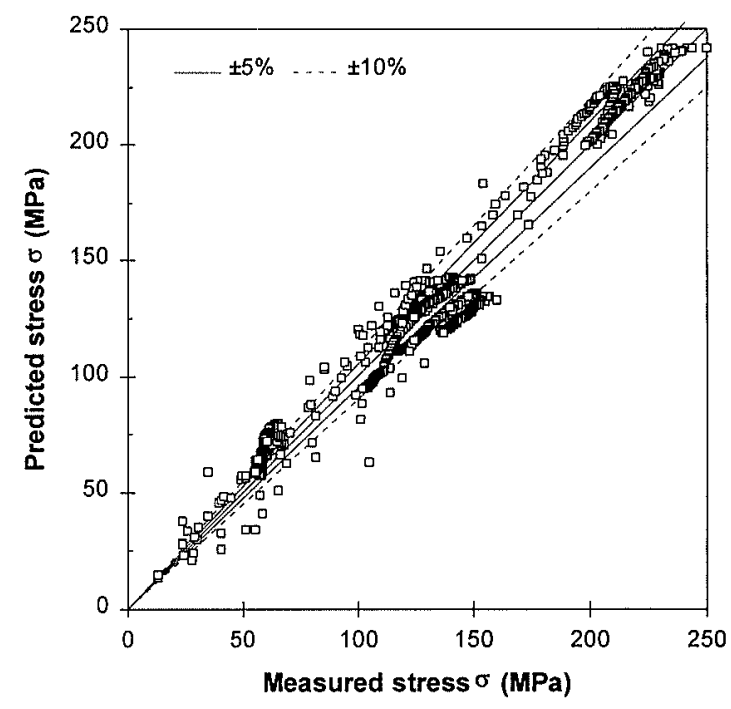

(b) Different steels but same strain rates and temperatures

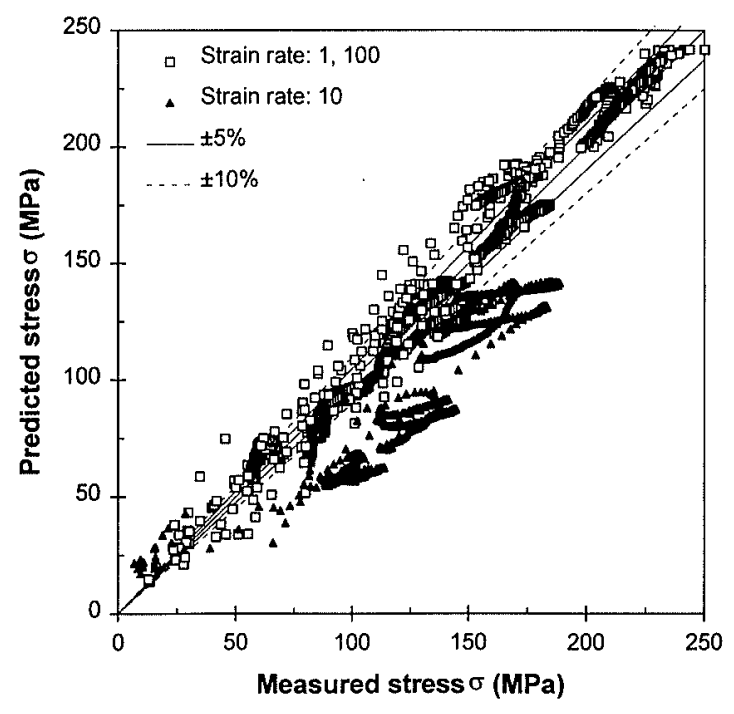

(c) Different steels, strain rates and temperatures

Fig. 11. Comparison of predicted and measured stress for test data (with boundary information only). 


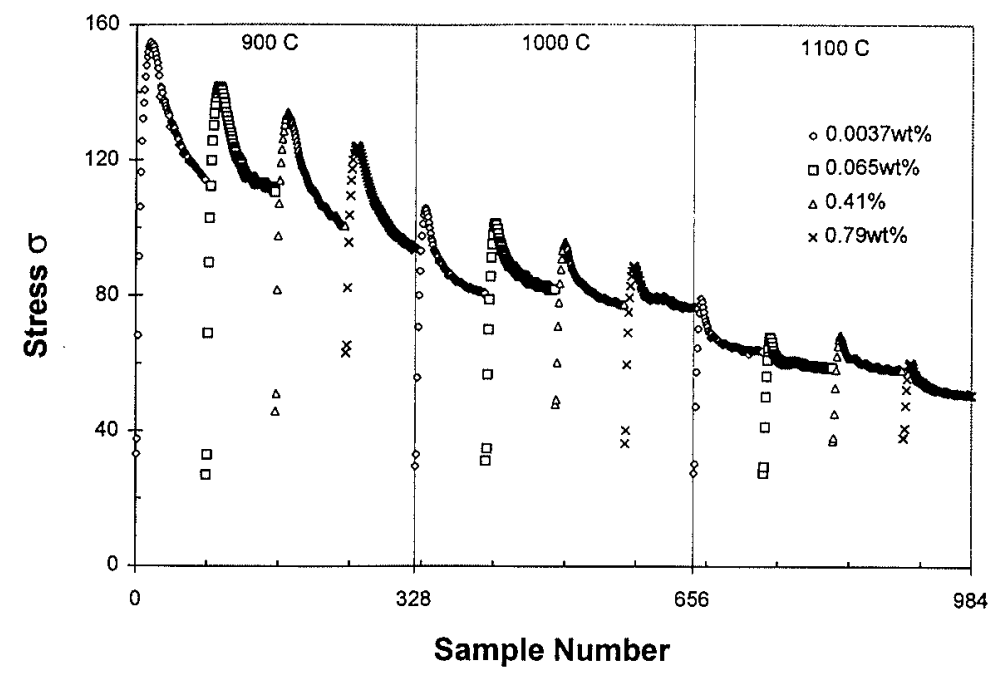

Fig. 12. Stress-strain curves for different steels at various temperatures. $\left(\dot{\varepsilon}=1 \mathrm{~s}^{-1}\right)$

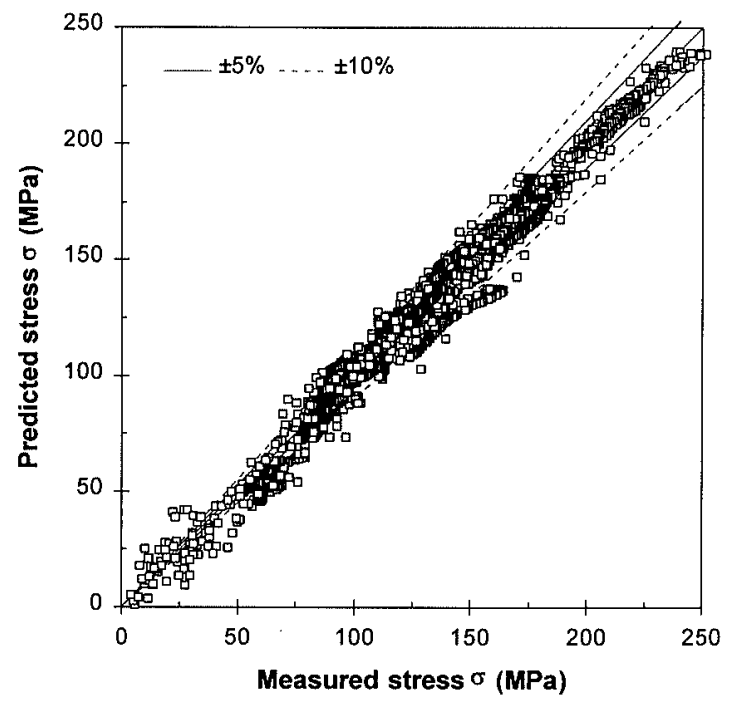

Fig. 13. Comparison of predicted and measured stress for training data (with boundary and intermediate information).

The prediction on steels B, D and F is shown in Fig. 14 with an average error of $4.4 \%$ and standard error of $8.8 \%$, which is much more accurate than the model with only boundary information provided for inputs of strain rate and temperature shown in Fig. 11(c). Apart from test at low $Z$ (at low strain rates and high temperatures) where the stresses are low, the modified ANN model accurately predicts the stress-strain behaviour in both the work hardening and dynamic recrystallisation regimes. Although the peak stress and peak strain vary with carbon content in the complicate way shown in Figs. 3 and 4, the modified ANN model accurately predicts these characteristics. Figure 15 indicates this agreement for the test steels at a strain rate of $10 \mathrm{~s}^{-1}$.

Although the carbon equivalent was used and able in the current work to evaluate the contribution of chemical composition, the modified ANN model could not predict the effect of materials in addition to carbon with high variation accurately at some deformation conditions. There were two heats $G$ and I with manganese different from others. The most of the prediction is very accurate, however, the peak strain at high strain rate of $100 \mathrm{~s}^{-1}$

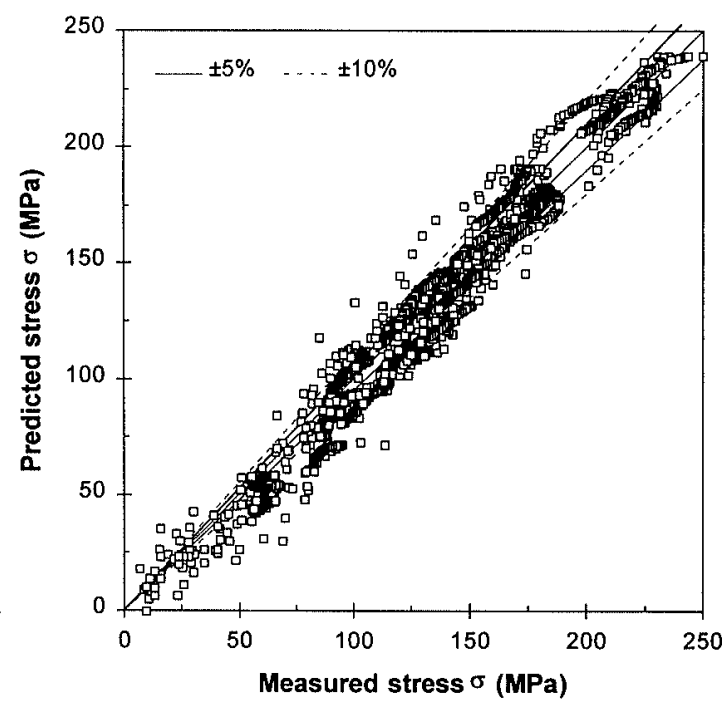

Fig. 14. Comparison of predicted and measured stress on test data of different steels (with boundary and intermediate information).

is not well predicted as carbon equivalent derived from high manganese with Eq. (3) shifts the peak strain to a lower value at high $Z$. In contrast to an irregular and complex effect of carbon content, manganese has an identical effect on hot strength as manganese has little effect on peak strain or slightly increase it under all conditions. In addition, there is a $4 \%$ increase in hot strength with an addition of $1 \%$ manganese regardless of deformation conditions. It would be expected that future modelling would need to incorporate the individual elements as inputs.

Comparing all of above modified ANN predictions with different training data, it is observed that appropriate model development and training data preparation can greatly improve the prediction accuracy and model generalisation. In all predictions with modified ANN models, the input, work hardening rate, developed from Kocks and Mecking model is the most sensitive parameter, the introduction of which greatly improves the prediction accuracy. The use of the Zener-Hollomon parameter as a further input also improves the prediction of peak stresses and peak strains. However, this requires 


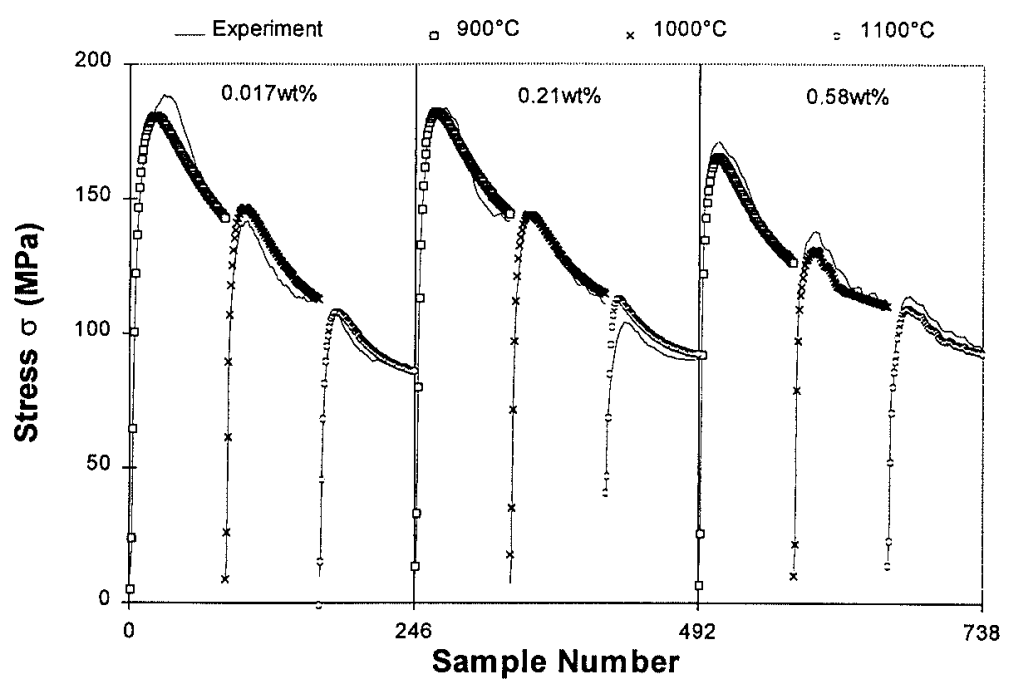

Fig. 15. Agreement between $A N N$ prediction and measurement at a strain rate of $10 \mathrm{~s}^{-1}$.

accurate knowledge of the activation energy, $Q_{\text {def }}$, which can be difficult to estimate if only a limited number of tests are performed. Although using less training data improves the accuracy for the training data, it reduces the capability of model generalisation. The training data needs to be prepared in accordance with the dependence of the stress-strain behaviour on the independent inputs to optimise the model accuracy, training efficiency and model generalisation.

\section{Conclusions}

The stress-strain behaviour of austenitic steels with carbon varying from 0.0037 to $0.79 \mathrm{wt} \%$ has been studied with a modified artificial neural network (ANN) model. The carbon content has a complex effect on flow strength of the austenitic steels studied in this work. An increase in carbon content reduces the flow stress of the steels at high temperatures and low strain rates, while it increases the flow stress at low temperatures and high strain rates, especially at low strains. In addition, increasing carbon above $0.4 \mathrm{wt} \%$ dramatically reduces the peak strain for the initiation of dynamic recrystallisation at high ZenerHollomon parameter, $Z$.

In this work, the back error propagation algorithm of the ANN model with one hidden layer bias was used, with the number of nodes of the hidden layer optimised for best performance. The effects of the carbon content on flow stress, peak strains and peak stresses observed from the experiment were accurately represented. However, the accuracy was highly dependent on the data used for training. If experimental data in a narrow range were used for training, higher accuracy of prediction could be achieved in this region. In order to more accurately predict the hot strength of the steels over a wide range, though an appropriate training data set should be provided for model generalisation. Deliberate removal of some data of critical carbon content at various temperatures and strain rates failed to predict the effect in this region due to the complexity of stress-strain behaviour varying with deformation conditions and composition. The training data should be prepared in accordance with the dependence of stress-strain behaviour on independent inputs to optimise the model accuracy, training efficiency and model generalisation.

\section{REFERENCES}

1) P. D. Hodgson, L. X. Kong and C. H. J. Davies: J. Mater. Process. Technol., (1998), accepted.

2) H. Mecking and U. F. Kocks: Acta Metall., 29 (1981), 1965.

3) Y. Estrin and H. Mecking: Acta Metall, 32 (1984), 57.

4) P. D. Hodgson: Australasia-Pacific Forum on Intelligent Processing and Manufacturing of Materials, Brisbane, Australia, (1997).

5) D. Ryan and H. J. McQueen: Mater. Sci. Technol., 81 (1986), 259.

6) Y. J. Hwu and J. G. Lenard: J. Eng. Mater. Technol., 100 (1988), 22.

7) Y. J. Hwu, Y. T. Pan and J. G. Lenard: Steel Res., 67 (1996), 59.

8) D. C. Collinson, P. D. Hodgson and B. A. Parker: Modelling of Metal Rolling Processes, IOM, London, (1993), 283.

9) D. C. Collinson, P. D. Hodgson and C. H. J. Davies: Thermec '97, Wollongong, Australia, (1997).

10) P. D. Hodgson, D. C. Collinson and B. A. Parker: Advances in Hot Deformation Textures and Microstructures, ed. by J. J. Jonas, T. R. Bieler and K. J. Bowman, TMS, Mass., (1993), 41.

1I) J. C. Principe, W. C. Lefebvre and N. R. Euliano: NeurSolutions Manual, (Version 2.1, 1994), vol. I.

12) A. Laasraoui and J. J. Jonas: Metall. Trans. A, 22A (1991), 1545.

13) U. F. Kocks: J. Eng. Mater. Technol., 98 (1976), 76. 